\title{
On the symmetric character of the thermal conductivity tensor
}

\author{
Ali R. Hadjesfandiari \\ Department of Mechanical and Aerospace Engineering \\ University at Buffalo, State University of New York \\ Buffalo, NY 14260 USA \\ ah@buffalo.edu
}

September 19, 2013

\begin{abstract}
In this paper, the symmetric character of the conductivity tensor for linear heterogeneous anisotropic material is established as the result of arguments from tensor analysis and linear algebra for Fourier's heat conduction. The non-singular nature of the conductivity tensor plays the fundamental role in establishing this statement.
\end{abstract}

\section{Introduction}

The conductivity tensor characterizes the general linear heat conduction relation between temperature gradients and heat flux in heterogeneous anisotropic material. By using nonequilibrium statistical mechanics, Onsager [1] has shown that the conductivity tensor is symmetric. However, classical continuum thermodynamics has not been able to provide any direct reasoning for this property, nor can it explain why we have to appeal to non-equilibrium statistical mechanics.

Here we establish the symmetric character of the conductivity tensor by using arguments from tensor analysis and linear algebra regarding the Fourier's heat conduction law. This includes results from the familiar eigenvalue problem concept and the theory of equations. Interestingly, the fundamental step in this establishment is based on the invertibility of the conductivity tensor. 
In the following section, we provide an overview of some important aspects ofelementary tensor analysis. This includes the definitions of tensors, their invariants and the character of the eigenvalues of second order tensors based on the theory of equations. In Section 3, we introduce the classical heat conduction relations for linear heterogeneous anisotropic material. After that in Section 4, the symmetric character of the conductivity tensor is established by using the arguments from tensor analysis and linear algebra. Finally, Section 5 contains a summary and some general conclusions.

\section{Preliminaries}

Consider the three dimensional orthogonal coordinate system $x_{1} x_{2} x_{3}$ as the reference frame, where $\mathbf{e}_{1}, \mathbf{e}_{2}$ and $\mathbf{e}_{3}$ are unit base vectors. This is the main coordinate system we use to represent the components of fundamental tensors and tensor equations. We also consider the primed orthogonal coordinate system $x_{1}^{\prime} x_{2}^{\prime} x_{3}^{\prime}$ for further investigation, having the same origin, but with $\mathbf{e}_{1}^{\prime}$, $\mathbf{e}_{2}^{\prime}$ and $\mathbf{e}_{3}^{\prime}$ as unit base vectors. The general orthogonal transformation between these systems is represented by the $3 \times 3$ transformation matrix $\left[a_{i j}\right]$, where

$$
a_{i m} a_{i n}=a_{m i} a_{n i}=\delta_{m n}
$$

Here the symbol $\delta_{i j}$ is the Kronecker delta. We notice that the components $a_{i j}$ are the direction cosines among the axes $x_{i}^{\prime}$ and $x_{j}$; that is

$$
a_{i j}=\cos \left(x_{i}^{\prime}, x_{j}\right)
$$

It should be noticed that the orthogonality relations (1) represents a set of six independent relations among the nine quantities $a_{i j}$. This shows that the orthogonal transformation matrix $\left[a_{i j}\right]$ is generally specified at most by 3 independent values.

The orthogonal transformations $a_{i j}$ among the different coordinate systems are essential in defining vectors and tensors based on the transformations of their components [2]. For example, 
the scalar $A$, vector $\mathbf{B}$ and second order tensor $\mathbf{C}$ transform such that their components in the primed coordinate system are

$$
\begin{gathered}
A^{\prime}=A \\
B_{i}^{\prime}=a_{i j} B_{j} \\
C_{i j}^{\prime}=a_{i p} a_{j q} C_{p q}
\end{gathered}
$$

It should be noticed that under orthogonal transformations some scalar values related to the components of $\mathbf{B}$ and $\mathbf{C}$ do not change, which are called invariants of these quantities. For the vector $\mathbf{B}$ there is only one invariant, which is the length $L_{B}$ of this vector. In terms of components, this invariant is

$$
L_{B}^{2}=B_{i} B_{i}
$$

For the second order tensor $\mathbf{C}$, its three eigenvalues are the three independent invariants. The eigenvalue problem is defined as

$$
C_{i j} v_{j}=\lambda v_{i}
$$

where the parameter $\lambda$ is the eigenvalue or principal value and the vector $\mathbf{v}$ is the eigenvector or principal direction. The eigenvalue problem (7) can be written as

$$
\left(C_{i j}-\lambda \delta_{i j}\right) v_{j}=0
$$

The condition for (8) to possess non-trivial solution for $v_{i}$ is

$$
\operatorname{det}\left(C_{i j}-\lambda \delta_{i j}\right)=0
$$

which in terms of elements can be written as

$$
\operatorname{det}\left[\begin{array}{ccc}
C_{11}-\lambda & C_{12} & C_{13} \\
C_{21} & C_{22}-\lambda & C_{23} \\
C_{31} & C_{32} & C_{33}-\lambda
\end{array}\right]=0
$$

This gives the cubic characteristic equation for $\lambda$ as

$$
\lambda^{3}-I_{C} \lambda^{2}+I I_{C} \lambda-I I I_{C}=0
$$

where the real coefficients $I_{C}, I I_{C}$ and $I I I_{C}$ are

$$
I_{C}=\operatorname{trace}(\mathbf{C})=C_{i i}
$$




$$
\begin{gathered}
I I_{C}=\frac{1}{2}\left[(\operatorname{trace} \mathbf{C})^{2}-\operatorname{trace}\left(\mathbf{C}^{2}\right)\right]=\frac{1}{2}\left[\left(C_{i i}\right)^{2}-C_{i j} C_{j i}\right] \\
I I I_{C}=\operatorname{det}(\mathbf{C})=\frac{1}{6} \varepsilon_{i j k} \varepsilon_{p q r} C_{i p} C_{j q} C_{k r}
\end{gathered}
$$

The symbol $\varepsilon_{i j k}$ in (14) is the alternating or Levi-Civita symbol.

Let us call the eigenvalues $\lambda_{1}, \lambda_{2}$ and $\lambda_{3}$. The cubic equation (12) with real coefficients has at least one real root. Therefore, in any case, one eigenvalue and its corresponding eigenvector are real, which we denote as the third eigensolution $\lambda_{3}$ and $\mathbf{v}^{(3)}$. We notice that the other two eigenvalues $\lambda_{1}$ and $\lambda_{2}$, and their corresponding eigenvectors $\mathbf{v}^{(1)}$ and $\mathbf{v}^{(2)}$ are either real or complex conjugate of each other. As a result, we can see that

$$
\begin{gathered}
I_{C}=\lambda_{1}+\lambda_{2}+\lambda_{3} \\
I I_{C}=\lambda_{1} \lambda_{2}+\lambda_{2} \lambda_{3}+\lambda_{3} \lambda_{1} \\
I I I_{C}=\lambda_{1} \lambda_{2} \lambda_{3}
\end{gathered}
$$

It should be mentioned that the vector $\mathbf{v}$ is usually normalized such that it becomes a unit vector, that is

$$
v_{i} \bar{v}_{i}=1
$$

where $\bar{v}_{i}$ is the complex conjugate of $v_{i}$.

Instead of the eigenvalues we may use their combinations $I_{C}, I I_{C}$ and $I I I_{C}$ as the new invariants, which can be expressed directly in terms of the elements of the tensor. Therefore, the real values $I_{C}, I I_{C}$ and $I I I_{C}$ are called the fundamental invariants of the tensor $\mathbf{C}$.

A second order tensor $\mathbf{P}$ is symmetric, if

$$
P_{i j}=P_{j i}
$$

The eigenvalues of the symmetric tensor $P_{i j}$ are all real and their corresponding eigenvectors are mutually orthogonal for distinct eigenvalues or can be taken mutually orthogonal for repeated 
eigenvalues. This means there is a primed orthogonal coordinate system $x_{1}^{\prime} x_{2}^{\prime} x_{3}^{\prime}$, where the representation of $P_{i j}^{\prime}$ is diagonal, that is

$$
\left[P_{i j}^{\prime}\right]=\left[\begin{array}{ccc}
P_{11}^{\prime} & 0 & 0 \\
0 & P_{22}^{\prime} & 0 \\
0 & 0 & P_{33}^{\prime}
\end{array}\right]
$$

A second order tensor $\mathbf{Q}$ is skew-symmetric, if

$$
Q_{i j}=-Q_{j i}
$$

It can be easily shown that the determinant of this tensor vanishes; that is

$$
I I I_{Q}=\operatorname{det}(\mathbf{Q})=0
$$

This in turn shows that at least one of the eigenvalues of the skew-symmetric tensor $Q_{i j}$ is zero.

We notice that the symmetry and skew-symmetry character of tensors are preserved in orthogonal transformations. Interestingly, the general second-order tensor $C_{i j}$ can be decomposed into the unique sum of its symmetric $C_{(i j)}$ and skew-symmetric $C_{[i j]}$ parts, such that

$$
C_{i j}=C_{(i j)}+C_{[i j]}
$$

where

$$
\begin{gathered}
C_{(i j)}=\frac{1}{2}\left(C_{i j}+C_{j i}\right)=C_{(j i)} \\
C_{[i j]}=\frac{1}{2}\left(C_{i j}-C_{j i}\right)=-C_{[j i]}
\end{gathered}
$$

Notice that here we have introduced parentheses surrounding a pair of indices to denote the symmetric part of a second order tensor, whereas square brackets are associated with the skewsymmetric part.

\section{Fundamental heat conduction theory}

Consider the heat conduction in a heterogeneous anisotropic solid material continuum at rest. In continuum mechanics, it is postulated that the amount of heat energy flow through a surface

element $d S$ with outward directed unit normal vector $n_{i}$ is $q_{n} d S$, where $q_{n}$ is the heat flux or 
thermal flux. Let us denote $q_{1}, q_{2}$ and $q_{3}$ as the heat fluxes through surfaces with unit normal in the direction of coordinate axes $x_{1}, x_{2}$ and $x_{3}$, respectively. It can be shown that these quantities define a heat flux vector $\mathbf{q}=q_{i} \mathbf{e}_{i}$ (see for example Carslaw and Jaeger [3]). As a result of this, we have the relation

$$
q_{n}=\mathbf{q} \bullet \mathbf{n}=q_{i} n_{i}
$$

for the heat flux $q_{n}$.

The combination of the first and second law of thermodynamics [2] results in the ClausiusDuhem inequality

$$
q_{i} T_{, i} \leq 0
$$

This inequality shows that the heat flux vector cannot have any positive component in the direction of temperature gradient.

\subsection{Linear heat conduction theory}

For linear heterogeneous anisotropic material, Duhamel's generalization of Fourier's heat conduction law [4] is

$$
q_{i}=-k_{i j} T_{, j}
$$

Here the tensor $\mathbf{k}$ is the material thermal conductivity tensor, which can vary from point to point. The minus sign in (25) assures that the heat flow occurs from a higher to a lower temperature. In terms of components, the conductivity tensor in the original coordinate system $x_{1} x_{2} x_{3}$ can be written as

$$
\left[k_{i j}\right]=\left[\begin{array}{lll}
k_{11} & k_{12} & k_{13} \\
k_{21} & k_{22} & k_{23} \\
k_{31} & k_{32} & k_{33}
\end{array}\right]
$$

Because it is required that the linear relation (28) be invertible, the conductivity tensor needs to be non-singular, that is

$$
\operatorname{det}(\mathbf{k})=\operatorname{det}\left[k_{i j}\right] \neq 0
$$


Since we have not established the symmetry character of $k_{i j}$, the nine components of $k_{i j}$ are independent of each other at this stage. Therefore, the conductivity tensor $k_{i j}$ is specified by nine independent components in the general case.

By decomposing the thermal conductivity tensor $k_{i j}$ into symmetric $k_{(i j)}$ and skew-symmetric $k_{[i j]}$ parts, we have

$$
k_{i j}=k_{(i j)}+k_{[i j]}
$$

where

$$
\begin{gathered}
k_{(i j)}=\frac{1}{2}\left(k_{i j}+k_{j i}\right)=k_{(j i)} \\
k_{[i j]}=\frac{1}{2}\left(k_{i j}-k_{j i}\right)=-k_{[j i]}
\end{gathered}
$$

In the general case, the tensors $k_{(i j)}$ and $k_{[i j]}$ are specified by six and three independent components, respectively.

By using the relation (28) for heat flux, we can write the Clausius-Duhem inequality (27) as

$$
k_{i j} T_{, i} T_{, j} \geq 0
$$

Since $k_{[i j]}$ is skew-symmetric, we have

$$
k_{[i j]} T_{, i} T_{, j}=0
$$

and

$$
k_{i j} T_{, i} T_{, j}=k_{(i j)} T_{, i} T_{, j}
$$

Therefore, the Clausius-Duhem inequality can be written as

$$
k_{(i j)} T_{, i} T_{, j} \geq 0
$$

which requires that the tensor $k_{(i j)}$ be positive definite. However, the Clausius-Duhem inequality does not impose any restriction on the tensor $k_{[i j]}$. In the following section, we prove that $k_{[i j]}$ 
vanishes based exclusively on tensor analysis. It should be emphasized that this proof is independent of the second law of thermodynamics and Clausius-Duhem inequality (27), which impose only the positive definite condition restriction on the symmetric part of the tensor $k_{(i j)}$.

\section{Symmetric character of the conductivity tensor}

Consider the heat conduction law in the original coordinate system $x_{1} x_{2} x_{3}$

$$
q_{i}=-k_{i j} T_{, j}
$$

Let us look for a direction of temperature gradient $T_{, i}$, which is parallel to the heat flux vector, that is

$$
q_{i}=-\lambda T_{, i}
$$

Therefore, by using (39) in (38), we obtain the eigenvalue problem

$$
k_{i j} T_{, j}=\lambda T_{, i}
$$

By considering the normalized unit vector $\mathbf{v}$ in the direction of the principal direction $T_{, i}$, we obtain the eigenvalue problem as

$$
k_{i j} v_{j}=\lambda v_{i}
$$

which can be written as

$$
\left(k_{i j}-\lambda \delta_{i j}\right) v_{j}=0
$$

Therefore, the condition for (42) to have a non-trivial solution for $v_{i}$ is

$$
\operatorname{det}\left(k_{i j}-\lambda \delta_{i j}\right)=0
$$

This is the characteristic equation for the tensor $k_{i j}$, which can also be written as

$$
\operatorname{det}\left[\begin{array}{ccc}
k_{11}-\lambda & k_{12} & k_{13} \\
k_{21} & k_{22}-\lambda & k_{23} \\
k_{31} & k_{32} & k_{33}-\lambda
\end{array}\right]=0
$$

As a result, the characteristic equation is the cubic equation 


$$
\lambda^{3}-I_{k} \lambda^{2}+I I_{k} \lambda-I I I_{k}=0
$$

where

$$
\begin{gathered}
I_{k}=\operatorname{trace}(\mathbf{k})=k_{i i} \\
I I_{k}=\frac{1}{2}\left[(\operatorname{trace} \mathbf{k})^{2}-\operatorname{trace}\left(\mathbf{k}^{2}\right)\right]=\frac{1}{2}\left[\left(k_{i i}\right)^{2}-k_{i j} k_{j i}\right] \\
I I I_{k}=\operatorname{det}(\mathbf{k})=\frac{1}{6} \varepsilon_{i j k} \varepsilon_{p q r} k_{i p} k_{j q} k_{k r}
\end{gathered}
$$

Since $\mathrm{III}_{k}$ is non-zero, all eigenvalues are non-zero. Therefore, the characteristic equation (45) has at least one real non-zero eigenvalue $\lambda_{3}$ with the corresponding real normalized eigenvector $\mathbf{v}^{(3)}$, where

$$
v_{i}^{(3)} v_{i}^{(3)}=1
$$

It should be mentioned that the relation (49) shows that the normalized eigenvector $\mathbf{v}^{(3)}$ is specified by two independent values in the original coordinate system $x_{1} x_{2} x_{3}$.

Now we choose the orthogonal coordinate system $x_{1}^{\prime} x_{2}^{\prime} x_{3}^{\prime}$ such that the axis $x_{3}^{\prime}$ coincides with the direction of this real unit eigenvector $\mathbf{v}^{(3)}$. Therefore, we have

$$
\left\{v_{i}^{\prime}\right\}^{(3)}=\left\{\begin{array}{l}
0 \\
0 \\
1
\end{array}\right\}
$$

Let us denote the plane normal to this direction as $\Pi$. In this plane, we choose the orthogonal axes $x_{1}^{\prime}$ and $x_{2}^{\prime}$ arbitrarily. The Fourier's heat conduction law in this special primed coordinate system $x_{1}^{\prime} x_{2}^{\prime} x_{3}^{\prime}$ becomes

$$
q_{i}^{\prime}=-k_{i j}^{\prime} T_{, j}^{\prime}
$$

As a result, for the eigenvalue problem (41) in this special primed coordinate system $x_{1}^{\prime} x_{2}^{\prime} x_{3}^{\prime}$, we have 


$$
k_{i j}^{\prime} v_{j}^{\prime}=\lambda v_{i}^{\prime}
$$

where the conductivity tensor is represented in the form

$$
\left[k_{i j}^{\prime}\right]=\left[\begin{array}{lll}
k_{11}^{\prime} & k_{12}^{\prime} & k_{13}^{\prime} \\
k_{21}^{\prime} & k_{22}^{\prime} & k_{23}^{\prime} \\
k_{31}^{\prime} & k_{32}^{\prime} & k_{33}^{\prime}
\end{array}\right]
$$

By examining the eigenvector (50) in the eigenvalue problem (52), we obtain

$$
\left\{\begin{array}{l}
k_{13}^{\prime} \\
k_{23}^{\prime} \\
k_{33}^{\prime}
\end{array}\right\}=\left\{\begin{array}{l}
0 \\
0 \\
\lambda_{3}
\end{array}\right\}
$$

As we can see, this relation requires that

$$
k_{13}^{\prime}=0, k_{23}^{\prime}=0, k_{33}^{\prime}=\lambda_{3}
$$

Therefore, the representation of the conductivity tensor in the special primed orthogonal coordinate system $x_{1}^{\prime} x_{2}^{\prime} x_{3}^{\prime}$ reduces to

$$
\left[k_{i j}^{\prime}\right]=\left[\begin{array}{lll}
k_{11}^{\prime} & k_{12}^{\prime} & 0 \\
k_{21}^{\prime} & k_{22}^{\prime} & 0 \\
k_{31}^{\prime} & k_{32}^{\prime} & k_{33}^{\prime}
\end{array}\right]
$$

Since the determinant of the conductivity is invariant, we have from (30)

$$
\operatorname{det}\left[k_{i j}^{\prime}\right]=k_{33}^{\prime} \operatorname{det}\left[\begin{array}{ll}
k_{11}^{\prime} & k_{12}^{\prime} \\
k_{21}^{\prime} & k_{22}^{\prime}
\end{array}\right] \neq 0
$$

This obviously requires

$$
k_{33}^{\prime}=\lambda_{3} \neq 0
$$

and

$$
\operatorname{det}\left[\begin{array}{ll}
k_{11}^{\prime} & k_{12}^{\prime} \\
k_{21}^{\prime} & k_{22}^{\prime}
\end{array}\right]=k_{11}^{\prime} k_{22}^{\prime}-k_{12}^{\prime} k_{21}^{\prime} \neq 0
$$

Now let us consider the temperature field, such that

$$
T=T^{\prime}=T^{\prime}\left(x_{1}^{\prime}, x_{2}^{\prime}\right)
$$

As a result, the relation (51) for the heat flux becomes 


$$
\begin{aligned}
& q_{1}^{\prime}=-k_{11}^{\prime} T_{, 1}^{\prime}-k_{12}^{\prime} T_{, 2}^{\prime} \\
& q_{2}^{\prime}=-k_{21}^{\prime} T_{, 1}^{\prime}-k_{22}^{\prime} T_{, 2}^{\prime} \\
& q_{3}^{\prime}=-k_{31}^{\prime} T_{, 1}^{\prime}-k_{32}^{\prime} T_{, 2}^{\prime}
\end{aligned}
$$

We notice that one should be able to obtain $T_{, 1}^{\prime}$ and $T_{, 2}^{\prime}$ for given heat flux $\left[q_{i}^{\prime}\right]=\left[\begin{array}{lll}q_{1}^{\prime} & q_{2}^{\prime} & q_{3}^{\prime}\end{array}\right]$. However, the system (61) for $T_{, 1}^{\prime}$ and $T_{, 2}^{\prime}$ is over-determined. Therefore, there must be a linear dependency among these equations.

By scrutinizing the relation (59), we realize that the first two equations

$$
\begin{aligned}
& q_{1}^{\prime}=-k_{11}^{\prime} T_{, 1}^{\prime}-k_{12}^{\prime} T_{, 2}^{\prime} \\
& q_{2}^{\prime}=-k_{21}^{\prime} T_{, 1}^{\prime}-k_{22}^{\prime} T_{, 2}^{\prime}
\end{aligned}
$$

are the required set of equations to obtain $T_{, 1}^{\prime}$ and $T_{, 2}^{\prime}$ for given heat flux components $q_{1}^{\prime}$ and $q_{2}^{\prime}$. As a result, the components of temperature gradient are explicitly expressed as

$$
\begin{aligned}
& T_{, 1}^{\prime}=\frac{1}{k_{11}^{\prime} k_{22}^{\prime}-k_{12}^{\prime} k_{21}^{\prime}}\left(-k_{22}^{\prime} q_{1}^{\prime}+k_{12}^{\prime} q_{2}^{\prime}\right) \\
& T_{, 2}^{\prime}=\frac{1}{k_{11}^{\prime} k_{22}^{\prime}-k_{12}^{\prime} k_{21}^{\prime}}\left(-k_{11}^{\prime} q_{2}^{\prime}+k_{21}^{\prime} q_{1}^{\prime}\right)
\end{aligned}
$$

From these relations, it is clearly seen that $T_{, 1}^{\prime}$ and $T_{, 2}^{\prime}$ are independent of the component $q_{3}^{\prime}$. Therefore, the last equation in (61) has to be trivially satisfied for any arbitrary given $q_{1}^{\prime}$ and $q_{2}^{\prime}$. This condition requires

$$
q_{3}^{\prime}=0, k_{31}^{\prime}=0, k_{32}^{\prime}=0
$$

As a result of this, the conductivity tensor is specified by five independent elements in the special primed coordinate system $x_{1}^{\prime} x_{2}^{\prime} x_{3}^{\prime}$; that is

$$
\left[k_{i j}^{\prime}\right]=\left[\begin{array}{ccc}
k_{11}^{\prime} & k_{12}^{\prime} & 0 \\
k_{21}^{\prime} & k_{22}^{\prime} & 0 \\
0 & 0 & k_{33}^{\prime}
\end{array}\right]
$$


The components of the conductivity tensor in the original unprimed coordinate system $x_{1} x_{2} x_{3}$ are obtained by using the transformation

$$
k_{i j}=a_{m i} a_{n j} k_{m n}^{\prime}
$$

where

$$
a_{3 i}=v_{i}^{(3)}
$$

or explicitly

$$
a_{31}=v_{1}^{(3)}, \quad a_{32}=v_{2}^{(3)}, \quad a_{33}=v_{3}^{(3)}
$$

Because of the normalizing condition (49), the relations in (68) enforce only two independent constraint values in (66). As a result, the conductivity tensor $k_{i j}$ is specified by seven independent elements in the original coordinate system $x_{1} x_{2} x_{3}$.

This result is in contradiction with our original statement that the conductivity tensor $k_{i j}$ is specified by nine independent components. To resolve this inconsistency, we consider the symmetric and skew-symmetric parts of the tensor $k_{i j}$. It is seen that the symmetric tensor $k_{(i j)}$ and skew-symmetric tensor $k_{[i j]}$ cannot be simultaneously specified by six and three independent components any more. For further investigation, we consider the three following possible cases:

Case (i). $k_{(i j)}$ and $k_{[i j]}$ are specified by four and three independent values, respectively. However, $k_{(i j)}$ is a general symmetric tensor with six independent values. This contradiction requires

$$
k_{(i j)}=0, k_{i j}=k_{[i j]}
$$

However, we notice that for this case

$$
\operatorname{det}\left[k_{i j}\right]=\operatorname{det}\left[k_{[i j]}\right]=0
$$

which violates the non-singularity condition for $k_{i j}$. This case is obviously not acceptable. 
Case (ii). $k_{(i j)}$ and $k_{[i j]}$ are specified by five and two independent values, respectively. This contradicts with the generality of the conductivity tensor. As a result, both tensor parts vanish; that is

$$
k_{i j}=k_{(i j)}=k_{[i j]}=0
$$

Therefore, this case also is not acceptable.

Case (iii). $k_{(i j)}$ and $k_{[i j]}$ are specified by six and one independent values, respectively. However, $k_{[i j]}$ is a general skew-symmetric tensor with three independent values. This contradiction requires

$$
k_{[i j]}=0
$$

Consequently, it is seen that this case is the only acceptable case, which states that the conductivity tensor is symmetric

$$
k_{i j}=k_{(i j)}
$$

This simply means

$$
k_{i j}=k_{j i}
$$

Therefore, the general conductivity tensor is specified by six independent components. Because of this symmetry character, the eigenvalues of this tensor are real and their corresponding eigenvectors are orthogonal. This shows that in our primed coordinate system $x_{1}^{\prime} x_{2}^{\prime} x_{3}^{\prime}$, the conductivity tensor given by (65) is specified by four independent elements such that

$$
k_{12}^{\prime}=k_{21}^{\prime}
$$

As a result, we can choose the axes $x_{1}^{\prime}$ and $x_{2}^{\prime}$ along the other orthogonal eigenvectors such that the conductivity tensor becomes diagonal, that is

$$
\left[k_{i j}^{\prime}\right]=\left[\begin{array}{ccc}
k_{11}^{\prime} & 0 & 0 \\
0 & k_{22}^{\prime} & 0 \\
0 & 0 & k_{33}^{\prime}
\end{array}\right]
$$


where, we have

$$
k_{11}^{\prime}=\lambda_{1}, \quad k_{22}^{\prime}=\lambda_{2}, \quad k_{33}^{\prime}=\lambda_{3}
$$

It is seen that the Clausius-Duhem inequality (37) can be written as

$$
k_{i j} T_{, i} T_{, j} \geq 0
$$

Since $k_{i j}$ is non-singular and symmetric, this inequality which requires that the tensor $k_{i j}$ be positive definite. This means that all eigenvalues (77) are positive.

As we mentioned before, the second law of thermodynamics and Clausius-Duhem inequality do not have any role here in establishing the symmetry character of the conductivity tensor. Our proof has been solely based on the tensorial character of quantities in Duhamel's generalization of Fourier's heat conduction law (28) by using some fundamentals of algebra.

\section{Conclusions}

By using arguments from tensor analysis and linear algebra, the symmetric character of the conductivity tensor for linear heterogeneous anisotropic material has been established. This shows that classical continuum mechanics can provide the mathematical reason for the symmetric character of the conductivity tensor, which is a necessary condition for having the consistent tensorial relations in classical heat conduction theory.

The method of proof here shows the subtle character of the tensors and their interrelationships, which has not been fully utilized in studying physical phenomena from this mathematical view. By using the character of tensor relations, we may find important results, which could not have been imagined previously in classical continuum mechanics.

Interestingly, the symmetric character of the resistivity tensor in Ohm's law for electric conduction and the diffusion coefficient tensor for Fick's law in mass transfer and other diffusive systems can be established using analogous methods. 


\section{References}

[1] L. Onsager, Reciprocal relations in irreversible processes. I, Phys. Rev. Lett. 37 (1931) 405-426.

[2] L. E. Malvern, Introduction to the Mechanics of a Continuous Medium, Prentice-Hall, Englewood Cliffs, New Jersey, 1969.

[3] H. S. Carslaw, J. C. Jaeger, Conduction of Heat in Solids, 2nd ed., Clarendon Press, Oxford, 1959.

[4] J.-M.-C. Duhamel, Sur les équations générales de la propagation de la chaleur dans les corps solides dont la conductibilité n'est pas la même dans tous les sens, J. Ec. Polytech. Paris, 13 (21) (1832) 356399. 